\title{
DESAFIOS À PESQUISA EM ENFERMAGEM
}

\author{
Challenges in nursing research
}

\author{
Desafíos a la investigación en enfermería
}

Lygia Paim $^{1}$

Mercedes Trentini²

Denise Guerreiro V. da Silva ${ }^{3}$

Arlete Arlinda Jochen ${ }^{4}$

\section{RESUMO}

0 artigo objetiva refletir acerca dos desafios da pesquisa inerentes às lacunas entre a teoria e a prática na área de enfermagem. Um olhar retrospectivo mostrou que a pesquisa em enfermagem continua sendo desenvolvida prioritariamente pelo Corpo Docente e Discente dos cursos de Pós-Graduação e que, na sua maioria, estas pesquisas têm produzido conhecimento teórico sem que se investigue o impacto na prática profissional. A não transportabilidade da produção do conhecimento teórico para os campos de atuação da prática de enfermagem requer o desenvolvimento de projetos de pesquisa focados na construção de tecnologias, estratégias e protocolos apropriados à incorporação de achados em investigações acumulados. Essa iniciativa de aplicabilidade significa que estes projetos requerem a inclusão dos saberes da prática.

Palavras-chave: Pesquisa em Enfermagem. Conhecimento. Prática Profissional.

\begin{abstract}
This paper aims to reflect on the nursing research challenges in relation to the gap between theory and practice. An overview of nursing publications shows that nursing research has been developed primarily by the nursing faculties and students of graduate programs. Those studies, however, have produced theoretic knowledge without important impact on the nursing practice. Therefore, the untried transference of the theoretic knowledge to nursing practice brings conflicts that require research projects focused on technologies, strategies and protocols for a proper application of the theoretic knowledge in nursing practice. This decision of applicability of theoretic knowledge means that such projects require also inclusion of the practice knowledge.
\end{abstract}

Keywords: Nursing Research. Knowledge. Professional Practice.

\section{Resumen}

El artigo objetiva reflexionar acerca de los desafíos de la investigación inherentes a las lagunas entre la teoría y la práctica en el área de enfermería. Una mirada retrospectiva mostró que la investigación en enfermería continua siendo desarrollada principalmente por los profesores e estudiantes de los cursos de Postgrados y que, en su mayoría, estas investigaciones han producido conocimiento teórico sin que se investigue el impacto que tiene en la práctica profesional. La intransferencia de la producción del conocimiento teórico en los campos de actuación de la práctica de enfermería requiere el desarrollo de proyectos de investigación centrados en la creación de tecnologías y el establecimiento de estrategias y protocolos adecuados para incorporar de resultados en investigaciones realizadas. Esta iniciativa de aplicabilidad significa que estos proyectos requieren de la inclusión de los saberes de la práctica.

Palabras clave: Investigación en Enfermería. Conocimiento. Práctica Profesional.

\footnotetext{
${ }_{1}$ Professora aposentada da Escola de Enfermagem Anna Nery da Universidade Federal do Rio de Janeiro. Professora do Curso de Graduação da Universidade do Vale do Itajaí/Campus de Biguaçu. Brasil. E-mail: lypaim@matrix.com.br, ${ }^{2}$ Professora aposentada do Departamento de Enfermagem e do Programa de Pós-Graduação em Enfermagem da Universidade Federal de Santa Catarina. Florianópolis/SC. Brasil. E-mail: mertini@terra.com.br, ${ }^{3}$ EProfessora do Departamento de Enfermagem e do Programa de Pós-Graduação em Enfermagem da Universidade Federal de Santa Catarina. Bolsista de produtividade do CNPq. Florianópolis/SC. Brasil. E-mail: denise@ccs.ufsc.br, ${ }^{4}$ Graduada em Ciências Econômicas e Especialista em Administração Hospitalar e Empreendimentos de Saúde. Assistente de Direção no Hospital Nereu Ramos. Florianópolis/SC. Brasil. E-mail: aajochen@bol.com.br.
} 


\section{INTRODUÇÃO}

Nos últimos trinta anos, a pesquisa na área da Enfermagem brasileira tem progredido tanto em quantidade quanto em qualidade, fato este evidenciado nas publicações dos periódicos de enfermagem e no registro de teses e dissertações no Centro de Pesquisa em Enfermagem - CEPEN. Essa explosão da pesquisa está associada à expansão e aperfeiçoamento dos cursos de mestrado e doutorado em enfermagem e da prática de pós-doutorado, e, por consequência, ao aumento também do número dos periódicos nesta área profissional.

Ao mesmo tempo em que se constata o crescimento da produtividade científica, emergem desafios à pesquisa de enfermagem. Reconhecemos que a prática da pesquisa ainda se restringe, em sua maior parte, às produções científicas realizadas pelo corpo docente e discente dos cursos de mestrado e doutorado com a elaboração das teses e dissertações. Resultados de um estudo que examinou pesquisas publicadas em algumas revistas de enfermagem sobre o tema Saúde do Trabalhador de Saúde constatou que, dos 114 autores, 50,8\% eram doutores, 3,5\% doutorandos, $1,8 \%$ livre-docentes, $8,8 \%$ mestres e 9,5\% mestrandos, perfazendo um total de $84,6 \%$. Todos esses tinham a docência como área de atuação. ${ }^{1}$ Outro estudo ${ }^{2}$ evidenciou que, de 14 publicações sobre a atenção à saúde do idoso, 6 eram de autoria de docentes e acadêmicos de pós-graduação; 5 , de docentes; e 3 , de docentes em coautoria de enfermeiros atuantes na prática.

Além dessas produções, os grupos de pesquisa se empenham na produção de investigações científicas, em parte para atender as exigências dos órgãos superiores como a CAPES e o CNPq. Essa exigência é positiva porque incita à produção, mas por outro lado, os professores, às vezes, são forçados a produzir e publicar trabalhos em número considerado suficiente por esses órgãos para serem avaliados produtivamente e nem todos dispõem de suporte logístico ou mesmo de conhecimento mais aprofundado sobre pesquisa para esta produção de maior complexidade. Assim sendo, muitas vezes a qualidade fica prejudicada, até porque a pesquisa científica há que sustentar qualidade e relevância, e estes dois atributos não podem ser negligenciados; do contrário, ela não se caracteriza científica.

Entre os pesquisadores de enfermagem, alguns se preocupam mais em ressaltar o tipo de metodologia e a precisão na implementação do método nas suas pesquisas do que, propriamente, destacar os achados referentes ao tema em pauta. 0 método é importante como meio para alcançar resultados em relação ao tema investigado, mas é a interpretação dos resultados que dá visibilidade e sentido às novas práticas no contexto estudado.

Quanto aos temas de pesquisa, vem se observando uma tendência de pesquisar sobre as atitudes dos usuários da saúde em relação a eles mesmos e em relação ao cuidado e a sua saúde. Esse tipo de informação vem se repetindo nos últimos anos nas pesquisas de enfermagem; contudo, mesmo obtendo essas importantes informações, pouco se sabe sobre o que a enfermagem vem fazendo na prática em resposta a esses achados detectados nas pesquisas. É preciso tomar cuidado para que o conhecimento desenvolvido nas pesquisas de enfermagem não "patine", pois, sem sua aplicabilidade na prática, o significado fica limitado, afetando os avanços, o que levaria à estagnação do conhecimento. A pesquisa é o apoio da prática e sem ela a prática também patina. ${ }^{3}$

As áreas do conhecimento, incluindo a da saúde, têm sido desafiadas com novas tecnologias e revigoradas para o enfrentamento das ações profissionais cotidianas. Será que a pesquisa da enfermagem brasileira está respondendo a esses novos desafios a fim de enfrentá-los na atenção à saúde dos usuários dos serviços de saúde? Há evidências de progresso do conhecimento na prática do cuidado de enfermagem? A prática de enfermagem apresenta indícios de articulação com 0 conhecimento decorrente das pesquisas e com a filosofia do Sistema Único de Saúde (SUS)? Estes questionamentos nos impeliram a refletir sobre os desafios da pesquisa e suas consequências na prática de enfermagem a partir da questão. Quão profícua tem sido a pesquisa em enfermagem na produção de novo conhecimento apropriado à canalização deste para a prática profissional, seja ela relacionada diretamente às atividades vinculadas ao cuidado em saúde, seja relacionada à formação educacional de novos profissionais?

\section{OS DESAFIOS DA PESQUISA EM ENFERMAGEM}

Por desafio da pesquisa em enfermagem entendemos um aglomerado temático o qual demanda projetos de pesquisa com enfoque na construção de novos conhecimentos, a fim de viabilizar a materialização do conhecimento abstrato no interior das práticas cotidianas do trabalho de enfermagem.

Muitos dos desafios da pesquisa em enfermagem inerentes à prática ao longo das últimas décadas têm sido, em parte, provocados pelo uso de referenciais mais universais, tidos como visões teóricas adaptadas à nossa cultura. Nos anos 60 e 70, com a adesão à abordagem holística, foram sendo valorizadas algumas teorias com ensaios de aplicação na prática profissional. Concepções teóricas, como as de: 
necessidades humanas básicas, interação, campos de energia, multidimensionalidade e transcendência, visão antropológica do cuidado, entre muitos outros ${ }^{4}$, vieram explicitando algumas tendências e influenciando a pesquisa. Embora esses referenciais tenham contribuído na mudança do modo de pensar a prática de enfermagem, o mesmo não tem ocorrido no alcance de investigações com descobertas voltadas às práticas cotidianas das atividades do cuidado e da educação de enfermagem. Por sua vez, algumas dessas teorias expressam mais as concepções das ações de enfermagem para o cuidado do que propriamente teorizar sobre o cuidado 5 .

Atualmente a atuação profissional em saúde está à frente de outros referenciais ético-legais e, consequentemente, também a novos desafios da pesquisa impulsionados pelo conhecimento de conceitos político-sociais, como participação e humanização, e os princípios da integralidade, universalidade e equidade. Essa constatação vem reavivar outros questionamentos: será que, mais uma vez, esse novo modo de praticar saúde permanece "estacionado" nos documentos próprios às resoluções governamentais? Será que o interesse em estudar as práticas de saúde está circunscrito aos trabalhos de conclusão de curso dos estudantes das Universidades sem atransposição do conhecimento para operar mudanças nos serviços de saúde?

A não transportabilidade da produção do conhecimento teórico para chegar aos campos de atuação da prática de enfermagem trazà cena variados conflitos de ordem epistemológica os quais desafiam a condução de outros projetos de pesquisa com enfoques na materialização do conhecimento até então produzido. 0 conteúdo teórico, isoladamente, tem sido mais valorizado pelos enfermeiros do que o aspecto de suatransposição paraa assistência de enfermagem, e isso os afastam do ser humano ${ }^{6}$, ao que é possível acrescentar que esta situação os afasta também das mudanças no contexto da prática.

A pesquisa de enfermagem, embora reconhecida formalmente por sua qualidade e relevância, ainda mostra um espaço não preenchido o qual se caracteriza como desencontro entre os achados investigativos e sua absorção no campo da prática profissional. Este desencontro é uma realidade desafiante embora se saiba que a intencionalidade dos pesquisadores seja a de vinculação de seus estudos ao cotidiano dessa prática profissional. Mesmo assim, ainda não se conseguiu o preenchimento dessa lacuna. Sejam quais forem as estratégias utilizadas, concordamos que a divulgação é o passo decisivo para a geração do impacto de qualquer estudo; não poderão existir mudanças enquanto não soubermos como mudar e são essas respostas que as pesquisas desenvolvidas pelos enfermeiros nos poderão fornecer. ${ }^{7}$

0 enfrentamento de tal situação vem sendo discurso recorrente na profissão e produz efeitos de superficial interpretação, quase sempre nos limites sociais comunitários sem, contudo, buscar esclarecer, em aprofundamento, a ordem epistemológica aí implicada.

A materialização dos achados das pesquisas de enfermagem não ocorre sem intencionalidade e decisão política. Esta decisão envolve o desenvolvimento de projetos apropriados à construção de maneiras protocolares, a fim de concretizar o conhecimento produzido na dimensão interior do cuidado e da educação em enfermagem. Este é um nó de natureza epistemológica e operacional que requer a construção de uma plataforma de estudos derivados de achados de pesquisas já produzidas. Com isto pode-se chegar a um forte sistema de tecnologias com valorização da comunicação bilateral: pesquisaprática profissional. A tendência profissional tem sido absorver como conhecimento convincente a mera assimilação teórica, pontilhada com alguns estágios. De certa forma, é preciso que os resultados das investigações cheguem às práticas e demonstrem congruência com a realidade dos cidadãos. ${ }^{8} \mathrm{Se}$ a decisão político-operacional do campo da investigação científica não ocorrer de modo a intensificar o preenchimento desta lacuna, construindo a ação resolutiva desse desencontro, a enfermagem estará fadada a permanecer nesse distanciamento das transformações em suas práticas cotidianas.

\section{SITUANDO OAGLOMERADO TEMÁTICO DAPESQUISA SOBRE O CUIDADO DA EDUCAÇÃO}

Em revisão por levantamento temático de teses nas classificações bibliográficas convencionais, foi possível visualizar, dentre 438 dessas teses, que a maior concentração esteve nos aglomerados temáticos denominados: cuidado e educação. 0 tema cuidado apresenta-se com 16,67\%, o que se amplia caso sejam incluídos os 4,02\% do que se costuma chamar de "educação em saúde", ou seja, práticas educativas inerentes à integralidade do cuidado em enfermagem. ${ }^{9}$ Embora o referido levantamento mostre que há um aglomerado temático coincidente com os interesses nas atividades de cuidado e educação de enfermagem, permanece 0 distanciamento entre os resultados dessas pesquisas e tais atividades. A lacuna, assim existente, implica um dos maiores desafios da investigação de enfermagem por ser esta uma disciplina que se fortalece em sua dimensão prática.

Na lógica da supressão de lacunas entre os resultados de pesquisa e a prática, há indícios de outras áreas e linhas de pesquisas que componham propositadamente um programa de transportabilidade dos achados investigativos face à realidade contextual de cada prática, como esquematizado na Figura 1. Isto fortaleceria a incorporação desses achados em práticas cotidianas da enfermagem, o que daria lugar a estudos destinados à construção 
de métodos, técnicas e estratégias configurando protocolos à organização do cuidado e dos serviços de saúde. A utilização de metodologia de pesquisa que trate de narrar e refletir a prática, tanto quanto desenhar estratégias especíicas de atenção à saúde, constituem "pontes" sobre as lacunas entre a teoria e a prática. ${ }^{10}$

Para a minimização das lacunas entre o conhecimento proveniente das pesquisas e a prática do cuidado de enfermagem há que se considerar que os resultados das pesquisas não são mecanicamente transportados como um novo conhecimento para serem absorvidos sem levar em conta as variáveis intervenientes das ações em determinado contexto de enfermagem profissional. Para isso, os profissionais atuantes na prática deveriam integrar os projetos de pesquisa; infelizmente, os enfermeiros e enfermeiras assistenciais não têm sido incentivados para tal atividade. 0 conhecimento de enfermagem vai além daquele expresso nas teorizações, mas também inclui aquele adquirido na experiência do exercício da prática profissional. ${ }^{11}$

A falta de integração das atividades acadêmicas e assistenciais tem contribuído nas limitações ao desenvolvimento da prática profissional e, consequentemente, na materialização da produção teórica pelas pesquisas. ${ }^{12} \mathrm{~A}$ fim de resolver os problemas inerentes à lacuna entre a teoria e a prática é necessário que os educadores instrumentem os estudantes a implementar 0 conhecimento teórico nas ações de cuidado de enfermagem. ${ }^{13}$

Para consubstanciar em modos tecnológicos 0 conhecimento construído pela investigação científica em suas bases teóricas, há o requerimento de capacidade profissional que significa a "intersecção inteligente entre teorizar práticas e praticar teorias" 8:29. Os pesquisadores de enfermagem precisam explicar como têm adaptado as metodologias derivadas das ciências sociais para a utilização no contexto da prática de enfermagem. Ao fazerem isso, não devem se preocupar com a aparente perda da pureza do método, mas precisam aceitar essa realidade enquanto mantêm rigor por meio da integridade, reflexibilidade, clareza de seus métodos e rítica construtiva do seu trabalho. ${ }^{14}$

Vale considerar que, no âmbito das práticas, a produção do conhecimento tem interfaces apreciáveis, mesmo ainda não tendo a investigação como proposta permanente no cotidiano dos serviços de saúde. Duas interfaces são fortes presenças nas práticas em serviços de saúde. 1) 0 clima de ensino-aprendizagem como busca natural de diferentes modos de fazer o cuidado em equipes de trabalhos, estágios, capacitações, construção de estudos para trabalhos de conclusão de curso - TCC, entre muitas formas de influência recíproca teoria-prática, no âmbito propício ao aprender fazendo. 2) Os caminhos deliberados de compreensão do instituído e das possibilidades instituíntes para a prática de ações afinadas com a construção do conhecimento sintonizado com o Sistema de Saúde.
Nessas interfaces da construção do conhecimento na prática, a base de construção do SUS desafia o próprio âmbito das pesquisas nos serviços de saúde para gerir o cuidado, retratando nele a versão da filosofia que o sustenta, dando materialidade a seus princípios de universalidade, integralidade e equidade. Isto se concretiza pela implementação de políticas de práticas de humanização em sua diretriz de acolhimento, de participação, de autonomia, cujos caminhos serão efetivados pelos saberes e fazeres e/ou modos tecnológicos que vão sendo construídos pelo aprender-fazendo. 0 referencial do Programa de Saúde da Família ${ }^{15}$ desafia para a desconstrução de um modelo de saúde superado e para a construção de práticas de saúde que induam vínculo, acolhimento, humanização e solidariedade. Também são essas interfaces da própria prática, um campo de saberes, no qual as investigaç̃os interagem por necessidade e convergência. A integração da teoria com a prática favorece a autoestima dos profissionais que atuam na assistência, pelo fato de se sentirem capacitados a expor a ancoragem de suas ações. Estudo desenvolvido com intuito de compreender a relação entre teoria e prática em enfermagem no sistema de informação no seu trabalho, concluiu, entre outras coisas, que, em nível social, os enfermeiros manifestaram o desejo de obter valorização da sociedade pela redução da lacuna entre a teoria e a prática no sistema de informação. ${ }^{16}$

\section{UM OLHAR PROSPECTIVO}

A solução para aproximar os achados de pesquisa de enfermagem da sua prática não se viabiliza unicamente por ideias, mas necessita de pesquisas que produzam no campo da prática novas tecnologias, estratégias e protocolos. Conquanto, no presente, esta questão implique em efeitos de lentidão e distanciamento entre as abstrações próprias do conhecimento teórico e a materialização desse conhecimento em tecnologias de práticas dele decorrentes, ainda assim é reconhecida a possibilidade de superar tal desencontro.

A construção de uma ponte que faça a síntese dessa diversidade de saberes requer mais do que uma compreensão linear que leve apenas os achados das pesquisas em blocos para que sejam referenciais ou explicações teóricas a qualquer prática. Muito mais que um bloco teórico explicativo, cada achado de investigação científica é, para a prática profissional, uma abstração a ser materializada em tecnologia correspondente às características e peculiaridades das práticas de enfermagem em determinadas situações. Entre um ambiente recortado metodológica e eticamente para a pesquisa e o contexto da prática cotidiana, há diferenças evidenciadas em interveniências fora do controle humano por ser imprevisível. Esta pode ser uma das 
principais fragilidades para que a pesquisa-prática no contexto da enfermagem se distinga da própria assistência, ao mesmo tempo em que estas incorporam e emitem conceitos, alimentando-se mutuamente.

Vencer esses impasses é passo que só se alcança com inserção do pesquisador no cenário das práticas cotidianas e isto requer que umaárea de investigação tenha como objeto de pesquisa a construção de tecnologias apropriadas à incorporação de achados já abstraídos de outras investigações. Isto significa dizer que esta área de pesquisa tem como lócus os serviços de saúde e valoriza os saberes de suas práticas em diálogo com os referidos achados de investigações pertinentes a determinados exercícios dos profissionais. Para esta situação se tornar viável é preciso haver maior integração entre as atividades de pesquisa e as da assistência.
Os profissionais de ambas as partes precisam adquirir capacidade de tomar o conhecimento construído nas pesquisas, como elemento principal da prática do cuidado de enfermagem. Além desse conhecimento formal, os projetos destinados a eliminar o vazio existente entre pesquisa e prática precisam levar em conta o saber adquirido pela experiência dos profissionais atuantes na prática. 0 maior desafio que se anuncia neste olhar em prospecção é o de desenvolver modos de composição da síntese desses saberese reverter tal síntese como elemento essencial da fisionomia tecnológica a ser incorporada, ou não, nas práticas correntes da enfermagem. Certamente isto requer uma política que acrescente um investimento maciço na capacitação dos profissionais para empreender a transportabilidade dos achados da pesquisa na prática.

Figura1. Esquema da transposição dos achados de pesquisa para a prática de enfermagem.

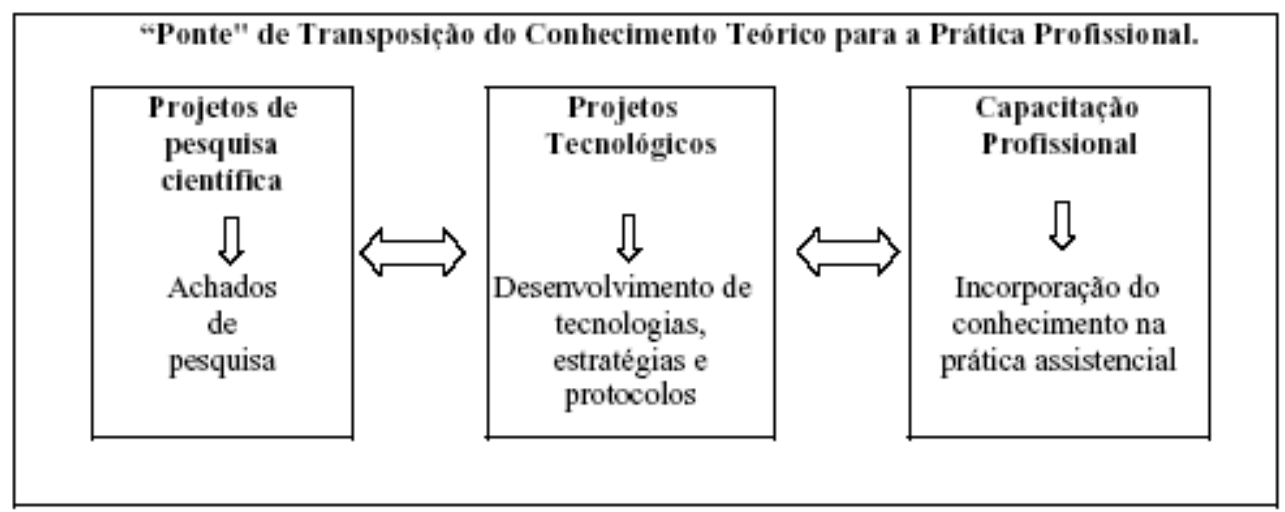

\section{REFERÊNCIAS}

1.Almeida VCF, Damasceno MMC, Araújo TL. Saúde do trabalhador de saúde: análise das pesquisas sobre o tema. Rev Bras Enferm 2005 maio/jun; 58(3): 335-40.

2.Veiga KCG, Menezes TMO. Produção do conhecimento em enfermagem: a (in) visibilidade da atenção à saúde do idoso. Rev Esc Enferm USP 2008; 42(4): 76176.

3. Ribeiro CM. A pesquisa e a prática da enfermagem. Anais do $3^{\circ}$ Seminário Nacional de Pesquisa em Enfermagem; 1984; Florianópolis (SC), Brasil. Florianópolis: Ed da UFSC; 1984. p. 10-26.

4.Meleis Al. Theoretical nursing: development and progress. $3^{\text {a }}$ ed. Philadelphia: Lippincott; 2004.

5.Almeida MCP, Rocha JSY. 0 saber de enfermagem e sua dimensão prática. $2^{\mathrm{a}}$ ed São Paulo (SP): Cortez; 1989.

6.Scherer ZAP, Luis MAV. Percepções e significados atribuídos pelos pacientes à vivência da queimadura. Acta Paul Enferm 1998 maio; 11(2): 64-72.

7.SantosZMSA, Oliveira VLM. A pesquisa de enfermagem e o impacto do conhecimento produzido Esc Anna Nery Rev Enferm 2003; 7 (2): 176-85.

8.Demo P. Pesquisa e construção de conhecimento: metodologia científica no caminho de Habermas. Rio de Janeiro (R): Tempo Brasileiro; 1994.

9. Trentini M, Paim L. Cenário atual da pesquisa de enfermagem brasileira. Congresso Criança 2005. $2^{\circ}$ Congresso Internacional de Especialidades Pediátricas - Módulo enfermagem; 30 ago 2005; Instituto de Ensino Superior Pequeno Príncipe. Curitiba (PR): Associação Hospitalar de Proteção à Criança Dr. Raul Carneiro; 2005.
10.Peden-McAlpine C. Using narrative and reflection to study nursing practice. First International Congress of Qualitative Inquiry; 2005 May 5-7; Illinois. Estados Unidos. Illinois: University of Illinois; 2005. Disponível em: http://mww.ilqi.org/C4Ql/httpdocs/ qi2005/Program1.pdf.

11.Santos SR. Sistema de informação em enfermagem: interação do conhecimento tácito-explícito. [on-line] Rev Bras Enferm 2005 58(1): 100-04.Disponível em: http://www.scielo.br/scielo.php?script=sci_arttext\&pid=S003471672005000100020\&lng=en.

12.Dyniewicz AM. Práticas educativas em metodologia de pesquisa para enfermeiras no contexto da clínica. Anais do $57^{\circ}$ Congresso Brasileiro de Enfermagem; 2005 nov. 03-07. Goiânia (GO), Brasil. Goiânia (GO): ABEn; 2005.

13.Scherer ZAP, Scherer EA. Reflexões sobre o ensino de enfermagem na pósmodernidade e a metáfora de uma lacuna teórico-prática. Rev Latino-am Enfermagem 2007 jun; 15(3): 498-501.

14.Gerrish K. Self and others: the rigor and ethics of insider ethnography. In: Latimer J. Advanced qualitative research for nursing. lowa(USA): Blackwell Publisching; 2003. p. 77-94.

15.Costa GD, Cotta RMM, Ferreira MLSM, Reis JR, Franceschin SCC. Saúde da familia: desafios no processo de reorientação do modelo assistencial. Rev Bras Enferm 2009 jan/fev; 62(1): 113-18.

16.Santos SR, Nóbrega MML. A busca da interação teoria e prática no sistema de informação em enfermagem: enfoque na teoria fundamentada nos dados Rev Latino-Am Enfermagem 2004 maio/jun; 12 (3): 460-68.

Data de recebimento: 26/05/2009 Data de reapresentação 08/10/2009 Data de aprovação 04/12/2009 